the inner cone, is screwed a light wooden beam, $f$, horizontally. Through the beam and lid passes a perforation for receiving the thermometer. At a little distance from the beam, $f$, and at the same height, there is a fixed pulley, $g$, over which is passed a cord with a scale at its pendant extremity, while the other end is attached to the end of one arm of the beam (the second arm of the beam. acts as counter weight). When the driving machine is put in action, the cones rub together, and the outer cone tends to carry the inner one and its beam round with it in the direction of rotation. With a certain weight in the scale, the horizontal part of the cord will form with the axis of the beam a right angle. From the length of the beam-arm, the amount of weighting, and the number of rotations, may be deduced the work that is transformed into heat; and from the water value of the calorimeter,

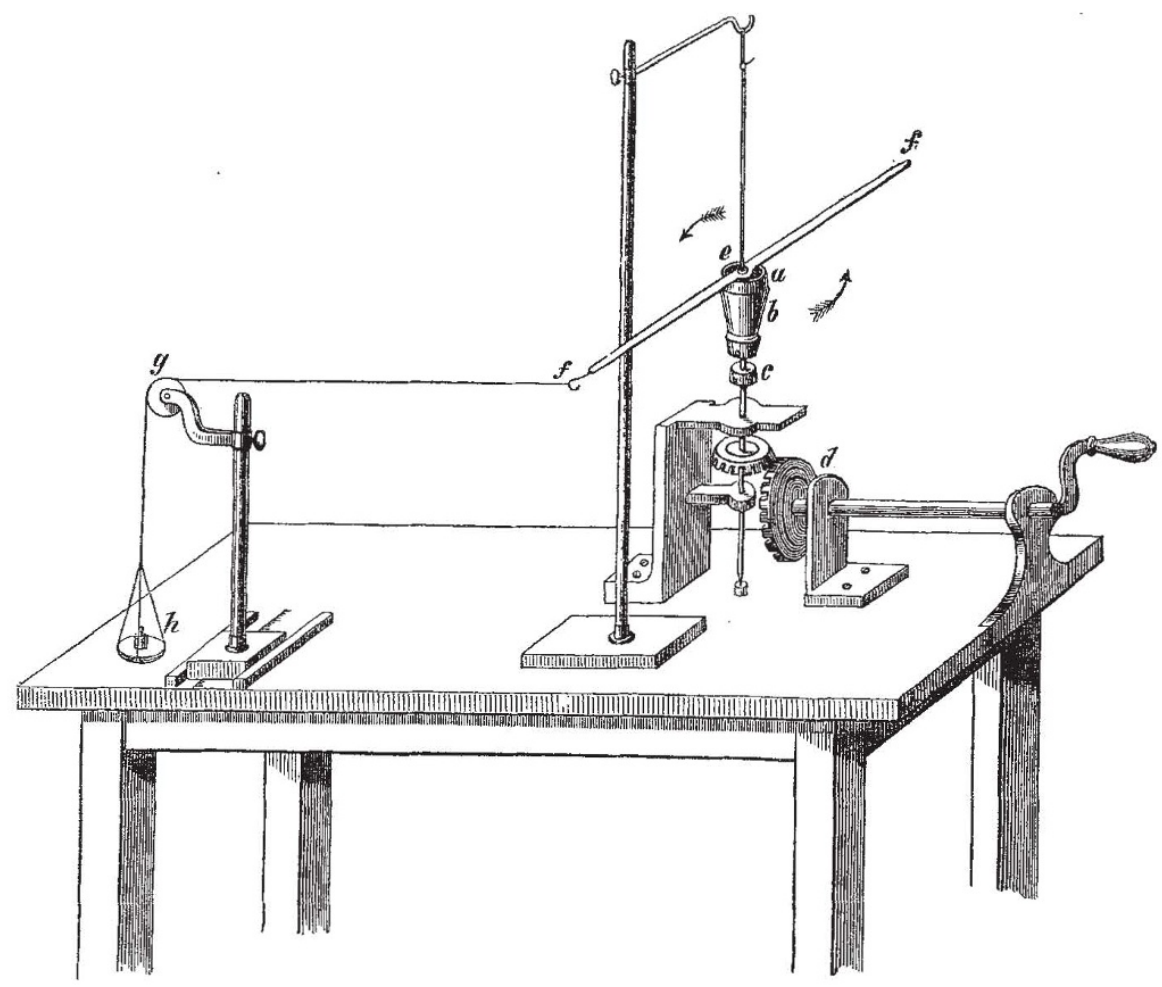

and the increase of its temperature, can be reckoned the quantity of heat produced.

From twenty-eight experiments (in which the amount of heat radiated from the calorimeter was taken into account), the average value obtained for the mechanical equivalent of heat was $425^{\circ} 2$, with a mean error $\pm 5^{\circ} 4$. A second series of experiments was made, with the arm of the beam in any position with reference to the cord. A simple arrangement-wooden triangle with arc-division - served for measuring the angle which the axis of the beam-arm formed with its normal position (in which it forms a right angle with the cord). From the observed values of this angle, and from the quantities already referred to, the number obtained for the mechanical equivalent of heat was $4^{\circ} 6^{\circ} 7$, with a mean error $\pm 5^{\circ} 9$.

The apparatus is especially to be recommended for lecture-experiments, because the method of experimenting is extremely simple, and the carrying out of the experiment takes very little time. A single experiment occupies 30-60".
S. W.

\section{THE PHYSICAL OBSERVATORY ON THE PIC DU MIDI}

$A \mathrm{~T}$ a recent sitting of the Paris Academy of Sciences A M. Ch. Sainte-Claire Deville made a communication, with reference to the proposed Physical Observatory on the Pic du Midi, in the Pyrenees. He referred to the increasing importance of meteorology, and to its manifold extensions and development in recent years, and to the growing necessity of establishing numerous fixed stations at as high an altitude as is practicable. This has already been done to a considerable extent in India, in America, and in some parts of Europe ; in France, as we have already intimated, the Puy de Dôme Observatory is nearly completed. M. Deville then referred to the importance of having a station on the Pyrenees, and to the difficulty of choosing a suitable site. The Pic du Midi de Bigorre, however, unites in itself all the most favourable circumstances. Situated towards the middle of the chain of the Pyrenees which receive directly the shock of the Atlantic storms, the Pic du Midi stands out from the general crest, and rises to a height of 2,877 metres, only 527 metres below the highest summit of the chain. It commands a magnificent and extensive panoramic view, and is easily accessible from various points. From the sixteenth century downwards it has attracted tee attention of men of science, and during the last and thpresent century a considerable number of notable ob servers have resorted to the Pic for the purpose of carrying on observations. Darcet, in 1786 , obtained from Philippe d'Orleans the promise of 80,000 francs to found an obser. vatory on the mountain, but the political events which rapidly succeeded prevented the scheme from being carried out. Even then a small hut existed on the spot where the Commission, charged by the Ramond Society with carrying out the present scheme, have built another; the former had been built by Vidal and Reboul, who in 1786-7 surveyed the Pic. Ramond, in the early part of 
the present century, made about thirty-six ascents of the peak, for the purpose of making barometric observations. In I 854 a society at Bagnères founded on the Col de Sencours, 5 I I feet below the Pic, on a hill immediately above the Lake Oncet, a hotel for tourists. It is this hotel which the Ramond Society has used as a temporary observatory, until the Government provide the means of erecting a proper building on the summit of the Pic. On August 1, 1873, the Commission appointed by the Society provided a tolerably complete set of meteorological apparatus on the Col de Sencours. Regular observations were carried on till October Io, when want of funds cut them short. On June I of the following year an observer, along with the President of the Commission, General de Nansouty, installed themselves and remained till December 25 , when, as we recorded at the time, the severity of the winter, for which they were insufficiently provided, compelled them to beat a precipitate retreat. On June I last year, General de Nansouty and M. Baylac again established themselves in the temporary observatory, and it is to be hoped they will be able to remain throughout the whole of the winter. An avalanche did considerable damage to the meteorological hut, and injured several of the instruments; fortunately, however, the observers managed to repair most of the damage done. The instruments which have been provided are of the best kind, and already observations of great value have been made, some of which have been published by the Ramond Society.

This Society determined to accomplish the erection of a proper observatory on the Pic du Midi itself, and has appealed to every quarter from which funds are likely to be obtained. The work of construction has already been begun. The building will be composed of three parts. The dwelling-house, situated seven metres below the summit, is in part subterranean, and will open only on the south side. It communicates by a tunnel with a circular vaulted erection, which will contain the barometer, the magnetic apparatus, \&c. At a little distance will be solidly built the Montsouris hut, intended to cover the instruments which must be subjected to the direct influence of the atmosphere.

The work is thus in progress, and there is every reason to believe that it will soon be successfully completed, and the station become one of the most important physical observatories, not only in France, but on the globe.

\section{PROF. TYNDALL ON GERMS*}

$7 \mathrm{HE}$ author refers in an introduction to an inquiry on the decomposition of vapours and the formation of actinic clouds by light, whereby he was led to experiment on the floating matter of the air. He refers to the experiments of Schwann, Schröder and Dusch, Schröder himself, to those of the illustrious French chemist Pasteur, to the reasoning of Lister and its experimental verification, regarding the filtering power of the lungs; from all of which he concluded, six years ago, that the power of developing life by the air, and its power of scattering light, would be found to go hand in hand. He thought the simple expedient of examining by means of a beam of light, while the eye was kept sensitive by darkness, the character of the medium in which their experiments were conducted, could not fail to be useful to workers in this field. But the method has not been much turned to account, and this year he thought it worth while to devote some time to the more complete demonstration of its utility.

He also wished to free his mind, and if possible the minds of others, from the uncertainty and confusion which now beset the doctrine of "spontaneous generation." Pasteur has pronounced it "a chimera," and expressed * On the Optical Deportment of the Atmosphere in reference to the Phenomena of Putrefaction and Infection. Abstract of a paper read before
the Royal Society, Jantary a th, by Prof, Tyndall, F.R.S. (Communicated
by the author.) the undoubting conviction that this being so it is possible to remove parasitic diseases from the earth. To the medical profession, therefore, and through them to humanity at large, this question is one of the last importance. But the state of medical opinion regarding it is not satisfactory. In a recent number of the British Medical Fournal, and in answer to the question, "In what way is contagium generated and communicated?" Messrs. Braidwood and Vacher reply that notwithstanding "an almost incalculable amount of patient labour, the actual results obtained, especially as regards the manner of generation of contagium, have been most disappointing. Observers are even yet at variance whether these minute particles, whose discovery we have just noticed, and other disease germs, are always produced from like bodies previously existing, or whether they do not, under certain favourable conditions, spring into existence de novo."

With a view to the possible diminution of the uncertainty thus described, the author submits without further preface to the Royal Society, and especially to those who study the etiology of disease, a description of the mode of procedure followed in this inquiry, and the results to which it has led.

A number of chambers, or cases, were constructed, each with a glass front, its top, bottom, back and sides being of wood. At the back is a little door which opens and closes on hinges, while into the sides are inserted two panes of glass, facing each other. The top is perforated in the middle by a hole 2 inches in diameter, closed air-tight by a sheet of india-rubber. This sheet is pierced in the middle by a pin, and through the pin-hole is passed the shank of a long pipette ending above in a small funnel. A circular tin collar 2 inches in diameter and $I \frac{1}{2}$ inch high, surrounds the pipette, the space between both being packed with cotton-wool moistened by glycerine. Thus the pipette, in moving up and down, is not only firmly clasped by the india-rubber, but it also passes through a stuffing box of sticky cotton-wool. The width of the aperture closed by the india-rubber secures the free lateral play of the lower end of the pipette. Into two other smaller apertures in the top of the case are inserted, air-tight, the open ends of two narrow tubes, intended to connect the interior space with the atmosphere. The tubes are bent several times up and down, so as to intercept and retain the particles carried by such feeble currents as changes of temperature might cause to set in between the outer and the inner air.

The bottom of the box is pierced with two rows, sometimes with a single row of apertures, in which are fixed air-tight, large test-tubes, intended to contain the liquid to be exposed to the action of the moteless air.

On Sept. Io the first case of this kind was closed. The passage of a concentrated beam across it through its two side windows then showed the air within it to be laden with floating matter. On the 13 th it was again examined. Before the beam entered, and after it quitted the case, its track was vivid in the air, but within the case it vanished. Three days of quiet sufficed to cause all the floating matter to be deposited on the sides and bottom, where it was retained by a coating of glycerine, with which the interior surface of the case had been purposely varnished. The test-tubes were then filled through the pipette, boiled for five minutes in a bath of brine or oil, and abandoned to the action of the moteless air. During ebullition aqueous vapour rose from the liquid into the chamber, vihere it was for the most part condensed, the uncondensed portion escaping, at a low temperature, through the bent tubes at the top. Before the brine was removed little stoppers of cotton-wool were inserted in the bent tubes, lest the entrance of the air into the cooling chamber should at first be forcible enough to carry motes along with it. As soon, however, as the ambient temperature was assumed by the air within the case, the cotton-wool stoppers were removed. 